\title{
Integration of complementary methods for monitoring stress/strain of wind turbine blades structures
}

\author{
Adriana Savin ${ }^{1, *}$, Rozina Steigmann ${ }^{1}$, Nicoleta Iftimie $^{1}$, Mariana Domnica Stanciu $^{2}$, \\ Narcis Andrei Danila ${ }^{1,3}$, and Paul-Doru Barsanescu ${ }^{3}$ \\ ${ }^{1}$ National Institute of R\&D for Technical Physics, NDT Department, Iasi, Romania \\ 2 Transilvania University of Braşov, Faculty of Mechanical Engineering, Brasov, Romania \\ ${ }^{3}$ Technical University “Gheorghe Asachi” of Iasi, Faculty of Mechanical Engineering, Iasi, Romania
}

\begin{abstract}
The most important part of wind turbine is the blade that must be tested during the fabrication and during the functioning when can be damaged by moisture absorption, fatigue, wind gusts or lightning strikes. The novelty of the paper is represented by the employing of wireless sensors embedded in a scalable wind turbine blade made from Glass Fiber Reinforced Plastics together with Fiber Bragg Gratings in order to effectuate structural health monitoring in static conditions. The sensors are placed on critical location on blade determined by FEM simulation and a comparison between the complementary methods is done.
\end{abstract}

\section{Introduction}

The EU is currently studying the possibility of updating the air pollution standards for power plants based on coal, because they are the largest source of emissions of sulfur and mercury dioxide, nitrogen oxides, arsenic, lead and cadmium. Thus, electrical power market obtained by harvesting wind power is expanding. In these conditions, to increase the power of turbines, wind turbines (WT) manufacturers use glass-fibers reinforced plastic (GFRP) for WT blades (WTB) allowing construction, due to low weight, of increased size blades. The monitoring of WTB made from GFRP have been reviewed in [1], proposing either statistical pattern recognition using simulation or experimental data, or in [2] considering mechanical property testing and full scale testing as well as nondestructive testing methods. Condition monitoring can be carried up using thermography [3] and fatigue crack detection performance can be done [4]. Implementing a process of fault detection and characterization of structures is refers as Structural Health Monitoring (SHM) [5]. The SHM process involves the observation of a system over time using periodically sampled dynamic response measurements from an arrangement of sensors, the extraction of damage-sensitive features from these features to determine the current state of system health [6]. In SHM domain, 7 axioms are accepted [7]. In our case, the most important are:

Axiom I: All materials have inherent flaws or defects.

\footnotetext{
*Corresponding author: asavin@phys-iasi.ro
} 
Axiom II: The assessment of damage requires a comparison between two system states. Axiom IVa: Sensors cannot measure damage. Feature extraction through signal processing and statistical classification is necessary to convert sensor data into damage information.

The nondestructive testing (NDT) techniques for in-service inspection and determination of regions with high degradation risks are developed, (function on the type and the size of the WT) [8]. In order to decrease the fabrication and maintenance costs, as well as for avoiding unproductive time, NDT is required both during fabrication and in-service.

In Romania, over 3\% of annual electrical power is produced by WT [9], the majority being GE1.5sle or similarly [10]. The most frequently flaws in turbine blades are microscopic and mesoscopic flaws, appeared in matrix, undetected by classical NDT procedures (as phased array ultrasound), broken fibers can appear and develop under moderate loading, cracks and delamination due to low energies impacts, etc. Starting from the scheme presented in Figure 1, flaws mentioned above must be detected and evaluated by NDT methods with good probability of detection (POD) under high reliability coefficient.

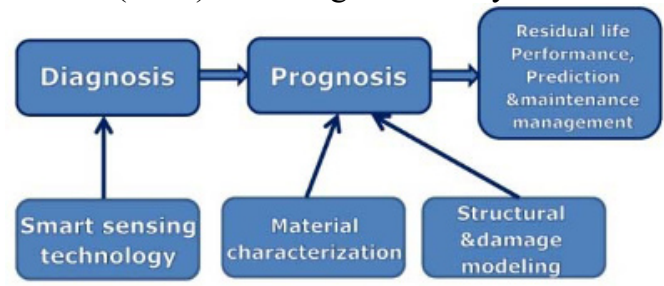

Fig. 1. SHM principle.

The paper presents the results of testing of a WTB, in static conditions, using wireless sensor (WRS) and Fiber Bragg Gratings (FBG) embedded within in order to monitor possible damages that further can be transformed into flaws. To determine the righteous of solutions, reliability of correct diagnosis probability, prognosis, and evaluation of residual lifetime and maintenance management, a scalable WTB has been constructed, that had the scaled dynamics of a full scale blade as well as enough sensors for measurements as well as monitoring. The maximum stress zones [11] and damage evolution and remnant stress [12] have been determined using Finite Element Modelling (FEM). The results of complex mechanical tests, effected on scalable WTB models (in our case a blade of $1750 \mathrm{~mm}$ length) are used to give efficiency to monitoring strategy.

\section{SHM based on transducers}

The SHM of WTB is characterized by sensors network around the blade to detect and locate any occurring damage. Taking into account the efficacy of the method and a priori knowledge about WTB, the sensors are distributed according to the most expected damage area, aiming the minimizing of the sensors number (Figure 2).

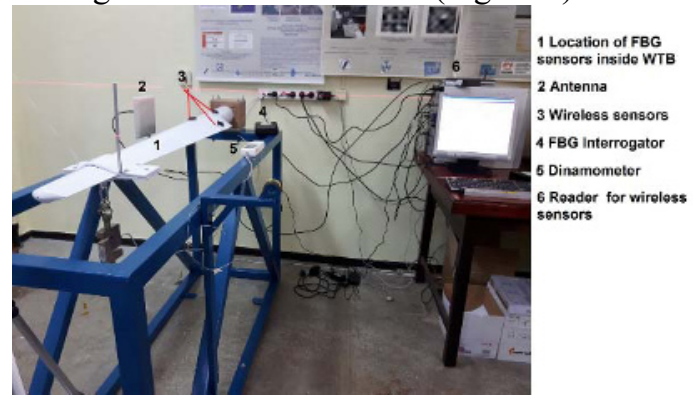

Fig. 2. Testing stand. 
The blade has been realized from E-glass/epoxy EPIKOTE Resin MGS LR 385 composite. The leading edge is straight and the trailing edge conical for an easy construction. The profile follows NACA airfoil. For increased structural strength and stiffness at $0.286 \mathrm{R}$ (R- the total length of distance between the center of rotor and the tip of blade), the same NACA has been applied both for the upper surface and lower surface, keeping the aerodynamically performances of the blade's tip. The profiles between $0.268 \mathrm{R}$ and tip were linear interpolated. A compromise has to be found between high-resolution and long propagation distance. The results are obtained at preliminarily testing of a scalable WTB, in laboratory, using optical fiber (OF) sensor technology and passive radiofrequency identification (RFID) WRS as uni-dimensional displacements sensor.

\subsection{Sensors with Fiber Bragg Gratings (FBG)}

SHM includes the use of FBG for detecting delamination in composite laminates [13] and monitor impact event occurrence [14]. [15]. The embedded sensor can monitories the structure in critical regions. The central wavelength of this signal, called Bragg wavelength $\lambda_{\mathrm{B}}$ is related to the physical parameters of the grating according to $\lambda_{B}=2 n \Lambda$ where $n$ is the effective refractive index of the fundamental mode propagating inside the fiber, $\Lambda$ is the pitch of FBG. When the fiber containing FBG is submitted to strain, the central wavelength is displaced to higher or smaller values [16]. The direction and the magnitude of displacement is proportional with the modification of strain or temperature. The strain axial sensitivity is

$$
\frac{1}{\lambda_{B}} \frac{d \lambda_{B}}{d \varepsilon}=\left(\frac{1}{n_{e f f}}\right) \frac{\partial n_{e f f}}{\partial \varepsilon}+\left(\frac{1}{\Lambda}\right) \frac{\partial n_{e f f}}{\partial \Lambda} \approx\left(1-p_{e}\right)
$$

where $\partial \lambda_{B}$ is the FBG wavelength shift; $\varepsilon<<1$, $\mathrm{p}_{\mathrm{e}}$ is photo-elastic coefficient of the fiber $p_{e} \approx 0.22$, FBG sensors whose gauge lengths are about $10 \mathrm{~mm}$ were used for SHM.

$$
\frac{\partial \lambda_{B}}{\lambda_{B}} \approx\left(1-p_{e}\right) \varepsilon ; \frac{\partial \lambda_{B}}{\lambda_{B}} \approx 0.78 \varepsilon
$$

The FBG strain sensing can be expressed as $\varepsilon=\frac{1}{0.78 \times 10^{-6}} \frac{\Delta \lambda_{B}}{\lambda_{B}}$.

FBG can assure and improve the functioning of WTB due to the detection properties, small dimensions and reduced weight, allowing the embedding them into the base material of the blade (being embedded at intersection of longeron with NACA profile, inside the WTB). In this structure, 3 FBG were embedded along the central longitude of the blade, placed into critical points determined by FEM of blade under vibration. Figure 3a presents the division of the blade in critical regions, in Figure $3 \mathrm{~b}$ is presented the result of simulation under a force $\mathrm{F}_{\mathrm{y}}=200 \mathrm{~N}$, producing the displacement of the tip with $1.59 \mathrm{~mm}$. The FEM model shown below takes into account the presence of the reinforcement structure, who's mass cannot be neglected, especially if one considers that the distance from the axis of rotation increase the inertia and can reduce the frequency associated with the first mode of vibration of WTB. Fortunately, the glass fibers employed makes the structure very rigid.
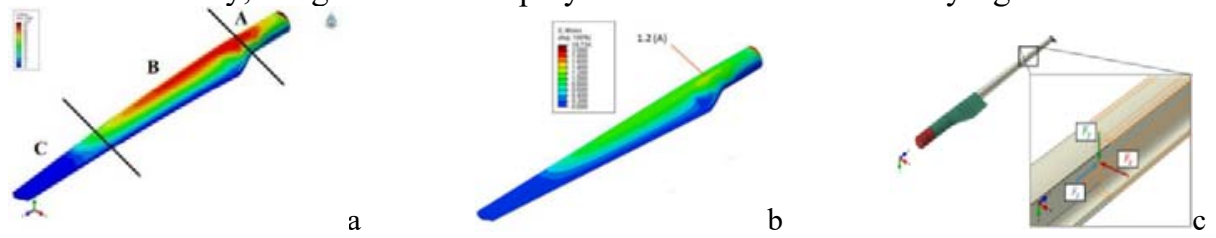

Fig. 3. FEM simulation of WTB: a) division of blade in critical regions; b) Von Mises stress; c) loading forces disposition. 
FBGs sensors were placed in region B at $307 \mathrm{~mm}, 362 \mathrm{~mm}$ and $406 \mathrm{~mm}$ from the hub fixing in stand. All the loadings are static. The WRS were placed in region with maximum critical points established by FEM.

\subsection{Wireless sensors (WRS)}

The passive WRS designed to monitory stress/strain status have as sensitive element a special type of metamaterial (MM), that follows the relative displacement (compression or tension) of its components due to an impact and can develop slows or either fast. The measurement of strain provides critical information on the force presented on material [17]. In specific construction as 2D geometry on flexible support, copper split ring resonators can be used as stress/strain sensors [18], [19]. In function of type and geometrical dimensions, the sensor has the resonance frequency in the range of RF and microwaves. The quality factor of the equivalent circuit has value in the range of tens or hundreds. MM [20] presents resonant properties [21] and around resonance frequency displays unusual properties. The inductance and the capacitance are given by [22]

$$
L=0.2 \mu_{0}\left(-\frac{w}{2} \sinh ^{-1} l+\frac{w}{2} \sqrt{2}+\left(l-\frac{w}{2}\right) \sinh ^{-1}\left(\frac{l-\frac{w}{2}}{\frac{w}{2}}\right)-\sqrt{\left(l-\frac{w}{2}\right)^{2}+\left(\frac{w}{2}\right)^{2}}\right) ; C=\varepsilon_{0} \varepsilon_{r} \frac{w_{1} t}{g}
$$

where $w_{l}$ is the distance between strips, $w$ is the width of strip, $l$ is the length of strip, $g$ is the gap and $t$ is the thickness of copper layer strip. The two circuit elements who constitute the sensor are considered as serial connected. The series resistances modifies the value of quality factor of the circuit element and, implicitly, the frequency band. The resonance frequency is

$$
f_{r}=\frac{c}{4(L+\Delta L) \sqrt{\varepsilon_{r}}}
$$

where $f_{r}$ is the estimated resonance frequency, $c$ is the speed of light in vacuum, $L$ is the length of the copper layer, $\varepsilon_{r}$ is the dielectric permittivity of the substrate, $\Delta \mathrm{L}$ is the additional length who compensate the effect due to thickness, width and dielectric constant of the substrate. For the developed sensor, the resonance frequency is $\mathrm{f}_{0}=834 \mathrm{MHz}$. Parallel with capacitance C, a capacitive element sensitive to stress/strain is connected, with the capacitance varying linear with the strain as $C_{\text {sensitive }}=\frac{\varepsilon_{r} \varepsilon_{0} w_{1} g}{u}\left(1+\varepsilon_{s}\right)$ where $u$ is the thickness of dielectric layer and $\varepsilon_{\mathrm{s}}$ is strain in $\mu \mathrm{m} / \mathrm{m}$. For the capacitive element, sensitive to stress/strain, to function upon a law closer to this, it is imposed that the Poisson ratio of dielectric support shall be as high as possible. For polyimide, the layer supporting copper strips, the Poisson ratio is 0.4 . If $\varepsilon_{\mathrm{s}}=10 \mu \mathrm{m} / \mathrm{m}$, the connection between the two capacitance make $\mathrm{C}$ increases with $10 \%$, inductance $\mathrm{L}$ remaining unchanged and the resonance frequency become $780 \mathrm{MHz}$. In order to obtain the best sensor with MM for monitoring the glass fiber composite structure, a sensor which allow the determination of resonance frequency displacement at the changing of dielectric properties from the sample has been developed. The system consist in a RFID reader and a RFID tag [11], the tag including the sensor and the integrate circuit. When the sensor detect a modification of the strain $\varepsilon$, the frequency is

$$
f_{r}^{\prime}=\frac{c}{4(1+\varepsilon)(L+\Delta L) \sqrt{\varepsilon_{r}}}=\frac{f_{r}}{1+\varepsilon} \approx f_{r}(1-\varepsilon)
$$

Thus, when the load is small, the resonance frequency modifies almost linear with load, the load can be determined if the resonance frequency is measured. The RFID tag antenna assures that the interrogation frequency $f$ shall be equal with the one of RFID tag to obtain 
perfect matching of impedance between antenna tag and IC chip. The smallest amount of energy must be transmitted toward reader to activate RFID tag, the transmitted power threshold (measured through the reader) reach minimum value at resonance frequency.

\section{Experimental results}

GFRP currently used in construction of WTB are usually long fiber-enforced composite materials. The fatigue test was performed with $50 \mathrm{kN}$ steps of tensile loading. The FBG are connected at optical system Spectral Eye 600 [23] coupled with PC via RS 232-USB2 connector. The data processing has been made in Matlab 2014b, the temperature correction being made, the equipment indicating the temperature of fiber, too. The central wavelength is $1527 \mathrm{~nm}$ and strain sensitivity $7.8 \times 10^{-7} \mu \varepsilon$. Figure 4a presents the raw data recorded by the device for different loadings. The relative variation of Bragg wavelength was determined in function of loading is presented in Figure $4 \mathrm{~b}$ showing that the relative variation of Bragg wavelength is linear. The dependency strain-load for WTB in a loading-unloading cycle for three sets of experimental measurement is presented in Figure 5. The same linear dependency strain-load can be shown, even the existence of a remnant stress at force removal, indicating an accumulation of energy in WTB composite structure, preponderant in the resin.
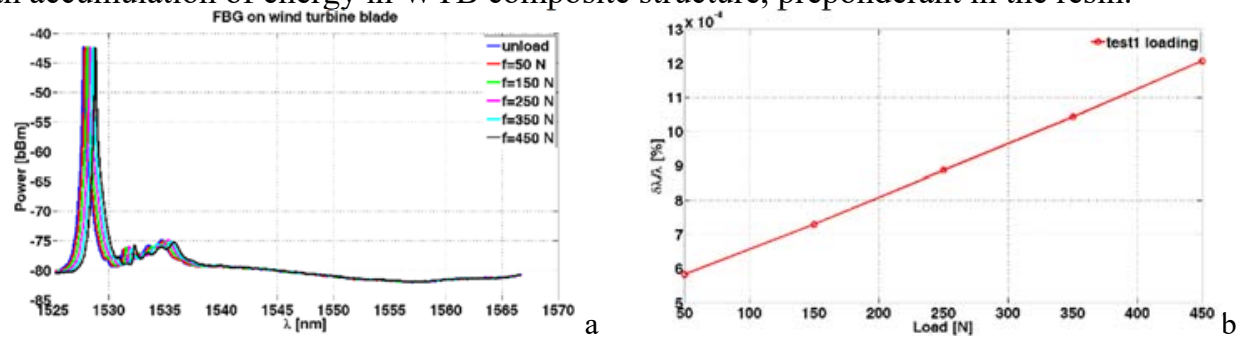

Fig. 4. FBG measurements: a) response to successive loading; b) relative variation of Bragg wavelength.

These stresses are relieving in relatively short time once with the material relaxation, so that the new tests are not influenced. The measurements using WRS were carried on the same time with those using OF sensors. The reader antenna (Figure 2) is fixed on the upper part of the stand, at $30 \mathrm{~cm}$ from the middle area of WRS placement locus. The antenna is connected to a logger reader coupled to PC by USB. The loading force is configured to have an increment of $243 \mu \varepsilon$ at each step. The mean threshold of interrogation power, in $\mathrm{dBm}$, is read by logger reader at each loading step. Figure 6 plots the experimentally dependency strainload for WTB into a cycle loading for the three WRS placed in different critical regions.

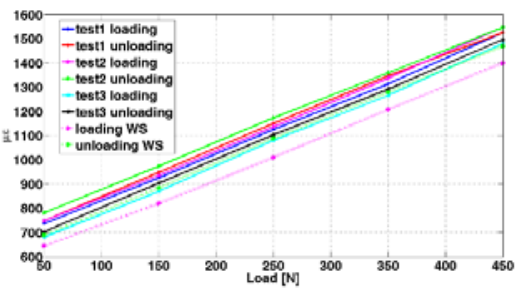

Fig. 5. The dependence of strain vs. loadings.

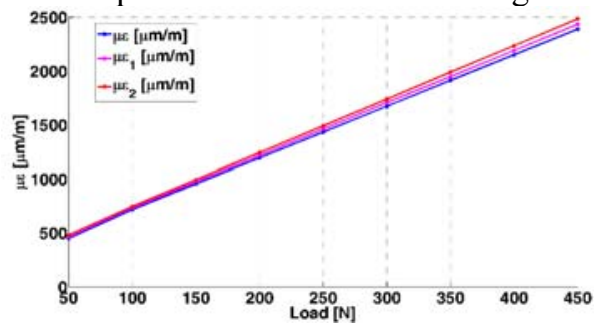

Fig. 6. Signal delivered by the 3 wireless sensors for loading of WTB.

It can be observed that the strain has a practically linear dependency on to the applied load on all WRS with small difference on slopes. Although the sensors are not located at exactly the same location, a great resemblance has been found. The measurement results of 
the FBG interrogation system was in excellent agreement with those obtained by wireless sensors measurements. The FBG sensor system and the wireless sensors offer many advantages over traditional resistive strain gauges. These include remote sensing, easy installation, non-corrosive and lower maintenance cost. This shows that complementary technologies are a good alternative for structural strain monitoring.

\section{Conclusions}

Monitoring complex structures as WTB has become a requirement. Practical applications demonstrates that, in order to avoid environmental disasters, it is necessary to establish diagnosis and prognosis methods, based on using information obtained from sensors constructed on known physical principles. The monitoring is close related with nondestructive evaluation and the trend is to obtain real time information. Scalable WTB have been constructed and tested to loadings using FBG and WRS embedded in the maximum concentration stress zones. The sensors responses obtained in static conditions were analyzed following a linear tendency, even they were not placed in the same regions. Both types of measurements shows advantages as remote sensing, simple embedding, and easy interpretation. The tests were carried on scalable models because in the frame of the project that sustains the paper, the blades will be employed into a demonstrator to show the righteous of solutions, reliability of correct diagnosis probability, prognosis, and evaluation of residual lifetime and maintenance management.

This paper is partially supported by Romanian Ministry of Research and Innovation under project PN-II-PCCA-2013-4-0656 and Nucleus Program PN 16-37 0101.

\section{References}

1. D. Adams, J. White, M. Ramsey, C.Farror, Wind Energy 14, 4 (2011)

2. B. Yang, D. Sun, Renew. Sust. Energ. Rev. 22 (2013)

3. B. Gao, W.L. Woo, Y. He, G.Y. Tian, IEEE T Ind. Inform. 12, 1 (2016)

4. S.G. Taylor, K. Farinholt, M. Choi, H. Jeong, J. Jang, G. Park, J.R. Lee, M.D. Todd, J. Intel. Mat. Syst. Str. 25, 5 (2014)

5. D. Balageas, C.P. Fritzen, A. Güemes, Structural health monitoring, 90, (John Wiley \& Sons, 2010)

6. C.R. Farrar, K. Worden, Philos. T. Roy. Soc. A. 365, 1851, (2007)

7. K. Worden, C.R. Farrar, G. Manson, G. Park, P. R. Soc. A, 463, 2082 (2007)

8. Wind turbine accident compilation, http://www.caithnesswindfarms.co.uk/fullaccidents.pdf

9. Wind Energy Targets for 2020 - 2030, EWEA (2011) http://www.ewea.org/fileadmin/files/library/publications/reports/Pure_Power_III.pdf

10. www.gerenewableenergy.com/wind-energy/turbines.html

11. R. Grimberg, G.Y. Tian, A. Savin, R. Steigmann, G.S. Dobrescu, Stud. Appl. Electromag. 39 (2014)

12. S. Antaya, Z. Ahmed, Eng. Fail. Anal. 35 (2013)

13. S. Takeda, Y. Aoki, Y. Nagao, Compos. Struct. 94 (2012)

14. K. S. C. Kuang, R. Kenny, M. P. Whelan, J. W. Cantwell, P. R. Chalker, Smart Mater. Struct. 10, 338 (2001)

15. J.M. López-Higuera, Handbook of Optical Fibre Sensing Technology, (Wiley and Sons: Chichester, West Sussex, UK, 2002). 
16. C.A.J. Gouveia, J.M. Baptista, P.A.S. Jorge, Current Developments in Optical Fiber Technology, Ch.13 (Dr. Sulaiman Wadi Harun Ed. 2013).

17. A. Deivasigamani, A. Daliri, Mod. Appl. Sci. 2 (2013)

18. T. Chen, S. Li, H. Sun, Sensors 12 (2012)

19. J.B. Pendry, A.J. Holden, D.J. Robbins, IEEE T. Microw. Theory Tech. 47, 11(1999)

20. D. Sievenpiper ; Lijun Zhang ; R.F.J. Broas ; N.G. Alexopolous ; E. Yablonovitch IEEE T. Microw Theory Tech., 47, 11, (1999)

21. J.B. Pendry, A.J. Holden, W.J. Stewart, I. Youngs, Phys. Rev. Lett., 76, (1996)

22. A. Savin, R. Steigmann, G.S. Dobrescu, 12th Biennial Conference on Engineering Systems Design and Analysis (ESDA2014) V002T07A027

23. FOS SpectralEye 600 Instruction Mannual, v.2.02, FOS\&S, Belgium 